\title{
A Escala de Personalidade Maquiavélica (MPS): Tradução e validação no contexto brasileiro
}

\author{
The Machiavellian Personality Scale (MPS): Validation in the Brazilian context
}

Márcia Zampieri Grohmann ${ }^{[a]}$, Luciana Flores Battistella ${ }^{[b]}$

\footnotetext{
${ }^{[a]}$ Doutorado em Engenharia de Produção pela Universidade Federal de Santa Catarina (UFSC), professor adjunto IV da Universidade Federal de Santa Maria (UFSM), Santa Maria, RS Brasil, e-mail: marciazg@gmail. com.br

${ }^{[b]}$ Doutorado em Engenharia de Produção pela Universidade Federal de Santa Catarina (UFSC), professora do Departamento de Ciências Administrativas da Universidade Federal de Santa Maria (UFSM), Santa Maria, RS - Brasil, e-mail: luttibattistella@gmail.com
}

Recebido: $11 / 12 / 2010$ Received: $12 / 11 / 2010$

Aprovado: 24/05/2011 Approved: 05/24/2011

\section{Resumo}

Este estudo trabalha com o maquiavelismo, mostrando suas relações com diversos temas organizacionais e buscando validar a Escala de Personalidade Maquiavélica - MPS (Dahling, Whitaker, Levy, 2009) para o contexto brasileiro. Dessa forma, foi realizada uma pesquisa descritiva, quantitativa, com 264 funcionários de uma mesma instituição divididos, de forma randômica, em duas partes: a primeira utilizada na análise fatorial exploratória (AFE), para purificar os dados; e a segunda na análise fatorial confirmatória (AFC), para a validação do modelo. A AFE gerou uma estrutura multifatorial (amoralidade, descrença, desejo de controle e desejo de status) com 19 variáveis e valor do KMO de 0,760, valor mínimo de alpha de Cronbach de 0,606 (fator status) e cargas fatoriais acima de 0,60. Na AFC os índices de ajustes encontrados foram: $\chi^{2}$ de 237,$262 ; \chi^{2}$ /gL de 1,77, estando dentro do intervalo aceitável; RMSEA de 0,057, estando abaixo dos 0,08 recomendados como limite máximo; GFI de 0,902, CFI de 0,860; NFI de 0,734 e NNFI de 0,840 , sendo que todos estes índices devem apresentar valores superiores a 0,90. Dessa forma, considera-se que o ajuste do modelo foi satisfatório, porém os resultados reforçaram a necessidade de novos estudos para validação da MPS, pois, além da redução de 45 para 19 itens, o modelo encontrado foi bem diferente do modelo original, validado por Dahling, Whitaker, Levy (2009).

Palavras-chave: Maquiavelismo. Organizações. Escala MPS. Validação. Fatorial confirmatória.

\section{Abstract}

Machiavellianism is the subject of this paper, which shows its relations with different aspects of organizational studies and seeks to validate the Machiavellian Personality Scale-MPS (Dahling, Whitaker; Levy, 2009) to Brazilian context. Thus, a quantitative research was made with 264 employees of an organization, and it was randomically split in two parts: the first part was used in Exploratory Factor Analysis (EFA) to purify data; the second part was used in Confirmatory Factor Analysis (CFA) to validate the model. EFA produced a multi-factor structure (amoral manipulation, distrust in others, desire for control and desire for status) with 19 variables and $K M O=0.760$, minimum Cronbach's Alpha $=0.606$ (status factor) and factorial loading above 0.60. CFA adjustment indices were: $\chi^{2}=237.262 ; \chi^{2} / g l=1.77 ; R M S E A=0.057 ; G F I=0.902 ; C F I=0.860$; $N F I=0.734 ; N N F I=0.840$, bearing in mind that all of those values should present values above 0.90 .

Psicol. Argum. 2012 jul./set., 30(70), 547-557 
This way, the model adjustment was satisfactory, although the results reinforced other studies are necessary to validate the MPS because, besides the decrease from 45 to 19 items, this model was found to present many differences from the original model proposed by Dahling, Whitaker, Levy (2009).

Keywords: Machiavellianism. Organizations. MPS Index. Validation. Confirmatory factorial.

\section{Introducão}

0 aumento do interesse sobre a responsabilidade socioambiental tem gerado uma séria de reflexos teóricos e práticos nas organizações. Observa-se um crescente número de artigos, linhas de pesquisas, cursos de pós-graduação e surgimento de periódicos e congressos específicos sobre o tema. Vários assuntos transversais também começam a ressurgir como, por exemplo, as questões éticas.

Diante deste cenário é que o presente estudo propõe a utilização de um construto vindo, originalmente da Psicologia, para os estudos organizacionais: o maquiavelismo. Para Dahling, Whitaker e Levy (2009) a revalorização da ética sugere que "o maquiavelismo possa ser um construto de interesse nos estudos organizacionais". Tang e Chen (2008) completam afirmando que ética e maquiavelismo possuem uma forte relação.

0 maquiavelismo tem diversas conexões com os estudos organizacionais e em outros países, principalmente na Europa e Estados Unidos, estão sendo divulgadas várias pesquisas sobre o tema (Deluga, 2001; Liu, 2008; Sakalaki, Kanellaki \& Richardson, 2009; Schepers, 2003; Shomenand \& Raon, 2009; Tang \& Chen, 2008), porém, no Brasil, essas relações ainda são incipientes.

Mas a questão principal para este estudo é o fato de que a maioria dos estudos realizados sobre maquiavelismo organizacional utiliza uma escala desenvolvida na década de 1960 e que possui problemas de validação estatística - a Mach IV. Dessa forma, este artigo testou uma nova escala de mensuração - a MPS - que subdivide o maquiavelismo em quatro construtos: amoralidade, desejo de controle, desejo de status e descrença nos outros.

Assim, o objetivo desta pesquisa é o de testar a escala MPS no contexto brasileiro, por meio da Análise Fatorial Confirmatória, buscando contribuir para o aprimoramento desta nova escala e para a sua divulgação, pois, pelas pesquisas realizadas, nenhum estudo publicado no Brasil já utilizou a MPS.
Para atingir seu objetivo, o artigo foi dividido da seguinte maneira: é apresentado o referencial teórico sobre maquiavelismo e estudos organizacionais e, principalmente, enfocando a escala MPS; a seguir apresentam-se os procedimentos metodológicos adotados, num terceiro momento estão os resultados encontrados, divididos em análises de estatística descritiva, análise fatorial exploratória, análise fatorial confirmatória e refinamento do modelo; e, por fim, as considerações finais, sugestões e limitações deste estudo.

\section{0 maquiavelismo e os estudos organizacionais}

Maquiavelismo, segundo Christie e Geis (1970, p. 106) pode ser definido como: "um processo no qual o manipulador obtém mais recompensa do que conseguiria sem manipulação, enquanto alguém consegue menos, pelo menos no contexto imediato" e sua origem são as ideias de Nicolau Maquiavel retratadas, principalmente, na obra O Príncipe (1982).

Pessoas com altos níveis de maquiavelismo são menos éticos e aceitam com mais facilidade comportamentos não éticos (Gunnthorsdottir, Mccabe \& Smith, 2002); têm maior predisposição a mentir e trapacear para obter vantagens (Ross \& Robertson, 2000); são capazes de sacrificar a ética, segundo seus interesses (Gable \& Dangelo, 1994); tem uma visão negativa das outras pessoas, mas são cínicos (Christie \& Geis, 1970); possuem grande capacidade de manipulação (Vlemming, 1979); são motivados por fatores externos, normalmente, aspectos materiais (Deci \& Ryan, 1985); e percebem as outras pessoas como ameaças (Vlemming, 1979).

Apesar dos maquiavélicos apresentarem algumas, ou todas, as características listadas anteriormente, várias estudos apontaram que para as organizações, em alguns momentos, em um número mais reduzido, ter funcionários maquiavélicos pode ser benéfico, pois, normalmente, seu desempenho é superior aos demais colegas (Gable \& Topol, 1991, 1998; Ricks \& 
Fraedrich, 1999). Ou seja, retoma-se a famosa frase de Machiavel "os fins justificam os meios".

Conforme destaca Vleeming (1979), quando o construto maquiavelismo começa a ser entendido também como uma conduta social e não apenas um traço de personalidade, é que começaram as associações entre maquiavelismo e organizações. As principais associações encontram-se no Quadro 1.

\section{A Escala de Personalidade Maquiavélica (MPS)}

Neste estudo, utiliza-se uma nova escala para mensuração do maquiavelismo, pois apesar da escala Mach IV, que até então era praticamente a única, ser amplamente utilizada em diversas pesquisas, os resultados destas são bem distintos, o que pode significar falhas na Mach IV (Ferh, Samson \& Paulhus, 1992). Essa escala foi desenvolvida por Christie e Geis (1970) e é dividida em três segmentos: táticas interpessoais, visão cínica da natureza humana e moralidade abstrata. Possui ao todo 20 itens que são afirmações que devem ser respondidas por meio de uma escala do tipo Likert. Os mesmos autores desenvolveram outra escala - Mach V - para complementar a anterior, composta por dez itens e que não utiliza a escala Likert, sendo que requer o uso de estatística não paramétrica (Zook, 1985).
Os principais problemas da Mach IV, segundo Dahling, Whitaker e Levy (2009), são:

- Dimensionalidade: apesar de Christie e Geis (1970) atribuírem três segmentos para a Mach IV, na verdade ela é uma escala unidimensional, além disso, um dos segmentos - moralidade abstrata - é representado por apenas dois itens, o que segundo Hair, Anderson, Tatham e Black (2005) prejudica as análises estatísticas confirmatórias. Dessa forma, "os resultados sugerem que a validade do construto da Mach IV é limitada e é necessária uma estrutura mais precisa de fatores" (Dahling, Whitaker \& Levy, 2009, p. 226).

- Poucos itens de escolha: além da escala ter poucos itens, apenas 20 para mensurar um construto tão complexo, a escala ainda apresenta vários itens que podem gerar comportamentos defensivos, como por exemplo: "pessoas que sofrem de doenças incuráveis devem ter a escolha de acabar com seu sofrimento, através da morte". Dessa forma, como salienta Spector (1992), as pessoas são induzidas a responder o politicamente correto.

- Validade inconsistente: os resultados obtidos a partir da Mach IV têm uma grande variabilidade

Quadro 1 - Efeitos do Maquiavelismo nas organizações

\begin{tabular}{|c|c|c|}
\hline Relação & Influência de Alto Nível e Maquiavelismo & Fontes \\
\hline Liderança & $\begin{array}{l}\text { Líderes diretos, adaptativos e carismáticos, isolados, } \\
\text { temidos e sem suporte dos subordinados. }\end{array}$ & Deluga (2001), Dory e Glusinkos (1980) \\
\hline Vantagem econômica & $\begin{array}{l}\text { Maximização de lucros e não compartilhamento dos } \\
\text { ganhos. }\end{array}$ & Sakalaki, Richardson e Thepaut (2007) \\
\hline Comportamento & $\begin{array}{l}\text { Tirar vantagens das oportunidades, violar a confian- } \\
\text { ça e não ajudar os outros. }\end{array}$ & $\begin{array}{l}\text { Fehr, Samson e Paulhus (1992), Harrel e Hart- } \\
\text { nagel (1976), Wolson (1981) }\end{array}$ \\
\hline Influências & $\begin{array}{l}\text { Construir relações políticas, atitudes de ingratidão e } \\
\text { intimidação. }\end{array}$ & $\begin{array}{l}\text { Dingler e Brown (1987), Harrel (1980); Pandey } \\
\text { e Rastogi (1979) }\end{array}$ \\
\hline Satisfação no trabalho & $\begin{array}{l}\text { Nunca estão satisfeitos com o trabalho ou com a } \\
\text { organização. }\end{array}$ & Gable e Topol (1987); Hunt e Chonko (1984) \\
\hline Vendas & São melhores vendedores. & Ricks, Fraedrich (1999), Ross, Robertson (2003) \\
\hline Políticas & São mais propensos a atitudes políticas. & Ferris et al. (2005) \\
\hline Confiança & $\begin{array}{l}\text { Maquiavélicos têm pouca propensão à confiança, o } \\
\text { que gera um clima de desconfiança na organização. }\end{array}$ & Mayer et al. (1995) \\
\hline Controle & $\begin{array}{l}\text { Organização com controle centralizado e com muito } \\
\text { pouca descentralização de responsabilidades. }\end{array}$ & Fehr et al. (1992); Murack (1989) \\
\hline
\end{tabular}

Fonte: Dados de pesquisa. 
e, o principal problema é o fato de que os valores obtidos pelo alpha de Cronbach são normalmente muito baixos. No estudo de White (1984) o valor encontrado foi de 0,46 e no de Geis e Moon (1981) foi de 0,52, sendo que para serem considerados satisfatórios os valores devem estar acima de 0,70 (Malhotra, 2001).

Dahling, Whitaker e Levy (2009) cientes dos problemas da escala anterior e, também, da sua grande contribuição para os estudos de maquiavelismo, utilizaram a Mach IV como base de sua nova escala: a Escala de Personalidade Machiavélica (MPS).

Porém a diferença crucial entre as duas escalas, segundo os autores, é que a MPS inclui dimensões do comportamento observável e também de crenças e motivações internas, pois o maquiavelismo é um construto complexo, formado por um conjunto de características. Assim, a proposta é a de se trabalhar com quatro construtos: manipulação amoral, descrença nos outros, desejo de status e desejo de controle, conforme demonstra o Quadro 2.

Dessa forma a escala MPS é composta de 45 itens, representados por afirmações na qual o entrevistado deve posicionar-se por meio de uma escala tipo Likert de 5 pontos (do discordo totalmente ao concordo totalmente), distribuídos em quatro construtos: 14 itens que refletem manipulação amoral;
11 itens que refletem desejo de controle; nove itens que refletem desejo de status; 11 itens que refletem descrença nos outros.

\section{Método}

Com o objetivo de validar, no Brasil, a escala MPS de mensuração do Maquiavellismo, foi realizada uma pesquisa descritiva e quantitativa (do tipo survey) com uma amostra inicial de 364 funcionários de uma mesma organização, distribuídos de forma aleatória entre todos os setores.

O nível de maquiavelismo foi testado por meio da escala desenvolvida por Dahling, Whitaker e Levy (2009). Assim, a coleta de dados foi realizada a partir de questionários compostos exclusivamente de questões fechadas que utilizam escala Likert de cinco pontos ( 1 = discordo totalmente; 5 = concordo totalmente), sendo 45 questões referentes à escala MPS e cinco questões que se relacionavam com características pessoais e profissionais dos respondentes. Conforme já mencionado, o modelo é formado por quatro construtos com 14 itens que refletem manipulação amoral, 11 itens que refletem desejo de controle, 9 itens que refletem desejo de status e 11 itens que refletem não confiança em outras pessoas.

Seguindo o procedimento proposto por Malhotra (2001), a escala foi traduzida para o português por

Quadro 2 - Construtos do Maquiavelismo na Escala MPS

\begin{tabular}{|c|c|c|}
\hline Construto & Definição & Explicação \\
\hline Descrença nos outros & $\begin{array}{l}\text { "Um olhar cínico sobre as motivações e intenções } \\
\text { das outras pessoas, com uma visão negativa das } \\
\text { implicações que essas intenções geram no self." }\end{array}$ & $\begin{array}{l}\text { Pessoas com alto nível de maquiavelismo procu- } \\
\text { ram manipular a situação e, dessa forma, acredi- } \\
\text { tam que os outros irão fazer a mesma coisa. }\end{array}$ \\
\hline Manipulação amoral & $\begin{array}{l}\text { "Propensão para romper padrões de moralidade e } \\
\text { valorizar comportamentos que beneficiam o self a } \\
\text { custa dos outros." }\end{array}$ & $\begin{array}{l}\text { Pessoas maquiavélicas são capazes de manipular } \\
\text { os outros, principalmente, de duas maneiras: } \\
\text { por meio do monitoramento e da administração } \\
\text { de impressões; e demonstrando mais flexibili- } \\
\text { dade quando tomam decisões. }\end{array}$ \\
\hline Desejo de status & $\begin{array}{l}\text { "Um desejo de acumular indicadores externos de } \\
\text { sucesso." }\end{array}$ & $\begin{array}{l}\text { Maquiavélicos são motivados por objetivos ex- } \\
\text { ternos e, principalmente, mensuram o sucesso } \\
\text { a partir do alcance desses objetivos externos } \\
\text { materiais. }\end{array}$ \\
\hline Desejo de controle & $\begin{array}{l}\text { “Desejo de controle é uma necessidade para } \\
\text { exercitar domínio sobre as situações interpessoais, } \\
\text { minimizando a extensão do poder dos outros.” }\end{array}$ & $\begin{array}{l}\text { Pessoas maquiavélicas consideram os outros } \\
\text { como ameaças e possuem o desejo de dominar } \\
\text { os outros e os acontecimentos. }\end{array}$ \\
\hline
\end{tabular}

Fonte: Dados da pesquisa. 
meio da técnica de tradução reversa e realizou-se um pré-testes (com dez funcionários) para a identificação de possíveis problemas. Esse procedimento demonstrou a necessidade de adaptação de alguns termos para uma linguagem mais informal.

Para que a validação de um modelo ocorra, a partir da Análise Fatorial Confirmatória, antes é necessária uma análise preliminar dos dados, por meio de estatísticas descritivas, análise fatorial exploratória e avaliação da confiabilidade e validade das escalas (Hair et al., 2005). Porém, conforme destacam Anderson, Gerbing e Hunter (1987), para que haja maior robustez e consistência à avaliação do instrumento, o banco de dados deve ser dividido em dois. Assim, de forma randômica, a amostra desta pesquisa foi dividida em duas partes: a primeira $(\mathrm{N}=182)$ foi utilizada para a análise fatorial exploratória e a segunda $(\mathrm{N}=182)$ para a análise fatorial confirmatória. Testes de diferença de médias demonstraram que não há diferenças significativas nos resultados das duas amostras.

\section{Resultados}

Os resultados serão apresentados de acordo com a seguinte sequência: uma breve caracterização da amostra e das médias dos construtos presentes na escala MPS; resultados da análise fatorial exploratória; resultados da análise fatorial confirmatória; e melhoria no modelo testado.

\section{Estatísticas descritivas}

Observa-se que a amostra foi composta de 199 pessoas do sexo masculino e 165 do sexo feminino, ou seja, $54,5 \%$ dos respondentes são homens e $45,3 \%$ são mulheres, destacando-se assim que a amostra foi bem equilibrada neste quesito. Em relação à idade, a maioria dos respondentes encontra-se na faixa de 31 a 40 anos e a menor frequência encontrada foi na faixa de menos de 25 anos, o que demonstra que os profissionais respondentes são adultos com mais de 30 anos de idade. A média de idade encontrada foi de 35,6 anos. Quanto ao tempo de atuação profissional, indo ao encontro dos dados anteriores, observa-se que a grande maioria dos profissionais entrevistados tem uma considerável experiência profissional, sendo que $22,4 \%$ possuem mais de 20 anos de trabalho, $27 \%$ de 15 a 20 anos e $28,8 \%$ entre 10 e 15 anos.
As médias finais dos construtos da escala MPS apontam que o desejo de status é a média mais elevada, com 3,1611 (desvio-padrão de 0,44746), em segundo lugar aparece a descrença nos outros, com 2,6879 (desvio-padrão de 0,52894), a seguir o desejo de controle com 2,2553 (desvio-padrão de 0,43467) e, por fim, amoralidade com 2,1123 (desvio-padrão de 0,40800). Nunnaly (apud Ricks \& Fraedrich, 1999), argumenta que se deva utilizar a média da escala (de 1 a 5) para determinar altos ou baixos níveis de maquiavelismo, assim, médias acima de 2,50 identificam um nível superior ao desejado para os construtos analisados. Dessa forma, observa-se que o desejo de status e a descrença nos outros se encontram em nível superiores aos esperados. Por outro lado, o desejo de controle e a amoralidade encontram-se abaixo do limite superior aceitável.

\section{Análise fatorial exploratória}

Nesta etapa, foi utilizada a técnica de análise fatorial que possui quatro etapas principais: cálculo da matriz de correlação, aplicação de rotação, adequação da aplicação da análise fatorial e extração dos fatores mais significativos (Malhotra, 2001). No presente trabalho utilizou-se a rotação varimax com o intuito de maximizar o peso de cada variável dentro de cada fator e como critério de extração foi definido autovalor maior que um.

A mensuração da adequação da aplicação da análise fatorial para um determinado conjunto de dados ocorre por meio de dois testes: esfericidade de Bartlett e Kaiser-Meyer-Olkin (KMO). 0 primeiro teste visa a identificar se a correlação entre as variáveis é significativa, a ponto de apenas alguns fatores poderem representar grande parte da variabilidade dos dados. Caso esse nível de significância seja próximo de zero, então, a aplicação da análise fatorial é adequada. 0 segundo teste (KMO) busca identificar se a correlação entre cada par de variáveis pode ser explicada pelas outras variáveis que formam o constructo. Valores iguais ou menores do que 0,5 indicam que a análise fatorial é insatisfatória.

Neste estudo, a estrutura fatorial final (Tabela 1) obteve valor do KMO de 0,760 e o valor do teste de esfericidade de Barlett foi de 182,752, com nível de significância de 0,000 . Valores que, segundo Pestana e Gageiro (2000) são considerados bons. Observa-se que as variáveis com comunalidades inferiores a 0,50 foram retiradas do modelo, por meio do método dos 
componentes principais e, desta forma, a escala MPS teve um número de variáveis reduzidas de 45 para 19 variáveis que foram divididas em cinco fatores. Todos os itens obtiveram valores de carga fatoriais acima de 0,60, com exceção da variável A7 $(0,587)$.

Tabela 1 - Análise fatorial exploratória

\begin{tabular}{|c|c|c|c|}
\hline & Item & $\begin{array}{l}\text { Carga } \\
\text { fatorial }\end{array}$ & Comunalidade \\
\hline & \multicolumn{3}{|c|}{ FATOR $1-$ Alpha Cronbach $=0,743$} \\
\hline $\mathrm{C} 01$ & $\begin{array}{l}\text { Eu acho que medo e } \\
\text { ameaças são coisas ne- } \\
\text { cessárias para motivar } \\
\text { as pessoas a fazerem o } \\
\text { que eu quero. }\end{array}$ & 0,688 & 0,473 \\
\hline $\mathrm{C} 08$ & $\begin{array}{l}\text { As ações de outras } \\
\text { pessoas constantemen- } \\
\text { te influenciam minhas } \\
\text { chances de sucesso. }\end{array}$ & 0,773 & 0,598 \\
\hline $\mathrm{C} 09$ & $\begin{array}{l}\text { Eu controlo o curso dos } \\
\text { eventos em minha vida. }\end{array}$ & 0,675 & 0,456 \\
\hline C10 & $\begin{array}{l}\text { Eu gosto de ter habili- } \\
\text { dade para controlar a } \\
\text { situação. }\end{array}$ & 0,676 & 0,457 \\
\hline \multirow[t]{2}{*}{ C11 } & $\begin{array}{l}\text { Eu determino o que } \\
\text { acontece na minha vida. }\end{array}$ & 0,706 & 0,498 \\
\hline & \multicolumn{3}{|c|}{ FATOR 2 - Alpha Cronbach $=0,606$} \\
\hline S03 & $\begin{array}{l}\text { Eu acredito que a maio- } \\
\text { ria das pessoas está fora } \\
\text { de seu próprio sucesso. }\end{array}$ & 0,775 & 0,601 \\
\hline S06 & $\begin{array}{l}\text { Eu quero ser rico e } \\
\text { poderoso algum dia. }\end{array}$ & 0,859 & 0,739 \\
\hline \multirow[t]{2}{*}{ S07 } & $\begin{array}{l}\text { O desenvolvimento } \\
\text { das pessoas é um } \\
\text { objetivo importante } \\
\text { para mim (I). }\end{array}$ & 0,694 & 0,482 \\
\hline & \multicolumn{3}{|c|}{ FATOR 3 - Alpha Cronbach $=0,689$} \\
\hline D07 & $\begin{array}{l}\text { Eu gosto de dividir } \\
\text { meus planos e ideias } \\
\text { com outras pessoas (I). }\end{array}$ & 0,753 & 0,570 \\
\hline D10 & $\begin{array}{l}\text { Se eu mostrar qualquer } \\
\text { fraqueza no trabalho, os } \\
\text { outros tirarão vanta- } \\
\text { gens disso. }\end{array}$ & 0,745 & 0,609 \\
\hline D09 & $\begin{array}{l}\text { Membros de equipe } \\
\text { puxam o tapete uns dos } \\
\text { outros para conseguir } \\
\text { destaque. }\end{array}$ & 0,739 & 0,581 \\
\hline D04 & $\begin{array}{l}\text { As pessoas só são mo- } \\
\text { tivadas por objetivos } \\
\text { pessoais. }\end{array}$ & 0,876 & 0,768 \\
\hline
\end{tabular}

Tabela 1 - Análise fatorial exploratória

(Conclusão)

\begin{tabular}{|c|c|c|c|}
\hline & Item & $\begin{array}{l}\text { Carga } \\
\text { fatorial }\end{array}$ & Comunalidade \\
\hline D03 & $\begin{array}{l}\text { Eu prefiro trabalhar } \\
\text { sozinho a precisar do } \\
\text { desempenho dos outros. }\end{array}$ & 0,799 & 0,696 \\
\hline \multirow[t]{2}{*}{ D06 } & $\begin{array}{l}\text { Quando tenho uma ideia } \\
\text { promissora, eu a mante- } \\
\text { nho comigo para evitar } \\
\text { que outros a roubem. }\end{array}$ & 0,637 & 0,586 \\
\hline & FATOR 4 - Alpha Cronbac & $=0,676$ & \\
\hline A08 & $\begin{array}{l}\text { Não há desculpas } \\
\text { para enganar outra } \\
\text { pessoa (I). }\end{array}$ & 0,840 & 0,709 \\
\hline A09 & $\begin{array}{l}\text { Eu estou disposto a ser } \\
\text { não ético se isso me } \\
\text { ajudar a ter sucesso. }\end{array}$ & 0,771 & 0,628 \\
\hline A07 & $\begin{array}{l}\text { Eu realmente só presto } \\
\text { atenção no que as pes- } \\
\text { soas dizem para desco- } \\
\text { brir se elas sabem algo } \\
\text { que pode me atingir. }\end{array}$ & 0,587 & 0,500 \\
\hline A10 & $\begin{array}{l}\text { Eu estou disposto a } \\
\text { sabotar os esforços dos } \\
\text { outros se isso estiver } \\
\text { prejudicando meus } \\
\text { objetivos. }\end{array}$ & 0,819 & 0,671 \\
\hline A06 & $\begin{array}{l}\text { A única boa razão para } \\
\text { falar com os outros é } \\
\text { conseguir informações } \\
\text { que eu possa usar para } \\
\text { me beneficiar. }\end{array}$ & 0,716 & 0,551 \\
\hline
\end{tabular}

Fonte: Dados da pesquisa.

Por fim, para avaliar a confiabilidade da escala, ou seja, a consistência interna entre os múltiplos indicadores de um construto, adotou-se o cálculo do coeficiente Alpha de Cronbach para cada uma das quatro dimensões encontradas (fatores). Os dados demonstraram os seguintes valores: controle - Alpha Cronbach $=0,743$; status - Alpha Cronbach = 0,606; descrença = 0,689; e amoralidade - Alpha Cronbach = 0,676. Estes índices são considerados satisfatórios, pois segundo Malhotra (2001) aceitam-se resultados acima de 0,60. Assim, conclui-se que o instrumento possui uma consistência interna satisfatória.

Assim, ao término da etapa da análise fatorial exploratória foi gerado um modelo hipotético que será testado com a análise fatorial confirmatória. Dos 45 itens do modelo original, restaram 19, sendo 
que cinco mensuram o construto amoralidade, seis a descrença nos outros, cinco o desejo de controle e três o desejo de status.

Comparando-se os resultados obtidos neste estudo com os encontrados por Dahling, Whitaker e Levy (2009), ver Tabela 2, identifica-se que a estrutura fatorial foi semelhante, ambos obtiveram quatro fatores, sendo que para um melhor ajuste, foram retirados vários itens, fornecendo, assim, um modelo com quatro fatores, perfazendo um total de 19 itens, o mesmo número da escala original. Os resultados de confiabilidade da escala, através dos alphas de Cronbach, encontrados nesta pesquisa foram inferiores aos do modelo original. Por fim, os resultados demonstram que as variáveis restantes após a análise fatorial foram bem diferentes nos dois estudos, o que demonstra uma divergência entre as realidades pesquisadas.

Tabela 2 - Índices comparativos das pesquisas

\begin{tabular}{lcc}
\hline Aspecto & $\begin{array}{l}\text { Modelo } \\
\text { original }\end{array}$ & Modelo encontrado \\
\hline Número de fatores & 4 & 4 \\
\hline Número de ítens & 19 & 19 \\
\hline $\begin{array}{l}\text { Alfa de Cronbach por } \\
\text { fator }\end{array}$ & 0,$83 ; 0,72 ;$ & 0,$68 ; 0,61 ; 0,74 ; 0,69$ \\
Variáveis de amora- & A3, A8, A9, & A6, A7, A8, A9, A10 \\
lidade & A10 e A11 & \\
\hline $\begin{array}{l}\text { Variáveis de controle } \\
\text { C2, C4, C10 }\end{array}$ & C1, C8, C9, C10, C11 \\
\hline $\begin{array}{l}\text { Variáveis de status } \\
\text { S1, S2, S8 }\end{array}$ & S3, S6, S7 \\
\hline $\begin{array}{l}\text { Variáveis de des- } \\
\text { crença }\end{array}$ & D4, D8, D9, & D3, D4, D6, D7, D9, D10 \\
\hline
\end{tabular}

Fonte: Dados da pesquisa.

\section{Análise Fatorial Confirmatória}

A Análise Fatorial Confirmatória (AFC) compreende uma série de procedimentos para que a validade de um modelo seja atestada. Nesta etapa, foi utilizada a segunda parte da amostra e, num primeiro momento, foi necessário excluir os dados omissos, pois seu percentual encontrava-se acima dos 5\% do total de dados. Dessa forma, a amostra ficou reduzida a 139 questionários, porém, como destacam Hair et al. (2005), os modelos, contendo até cinco construtos, cada um com no mínimo três itens de mensuração e com comunalidades acima de 0,60 , podem ser realizadas com amostras pequenas de 100 a 150 questionários. Como o modelo proposto, enquadra-se em todas estas três características, julgou-se o tamanho da amostra adequado para a realização da AFC.

Conforme foi demonstrado na AFE, a validade do construto foi comprovada, pois as cargas fatoriais todas acima de 0,60 identificaram que há validade convergente e os valores dos alphas de Cronbach (todos superiores a 0,60) atestaram a confiabilidade do construto. Porém, antes de submeter os dados a AFC, foi necessária a verificação de alguns pressupostos estatísticos. Para tanto, utilizou-se os procedimentos propostos por Lopes (2005) de identificação de: valores discrepantes; normalidade; linearidade; homogeneidade de variância e multicolinearidade.

Para a identificação de casos extremos foi utilizado o diagrama em caixa e como nenhum dos valores discrepantes situou-se a 1,5 ou mais desvios quartílicos - distância de mahalanobis - do quartil superior ou inferior (Lopes, 2005), comprovaram-se a ausência de casos extremos. Os gráficos Q-Q e P-P demonstraram que boa parte dos dados apresentavam distribuição normal, o que segundo Hair et al. (2005), é o pressuposto mais importante da AFC. Mas, para a comprovação da normalidade foi realizado o teste Kolmogorov-Smirnov (KS) que obteve níveis de significância de 0,000 para apenas três itens, demonstrando que a algumas distribuições não seguiam a normalidade. A linearidade e a multicolinearidade foram analisadas a partir da correlação de Pearson e os índices demonstraram que a maioria das correlações não foram significativas o que aponta que a não multicolinearidade é moderada.

A AFC foi realizada com a máxima verossimilhança para a estimação dos parâmetros e o teste de ajuste do modelo foi analisado por meio dos seguintes itens: estatística qui-quadrado $\left(\chi^{2}\right)$, qui-quadrado/graus de liberdade $\left(\chi^{2} / \mathrm{gl}\right)$ root mean square residual (RMR), root mean square error of aproximation (RMSEA), goodness off it índex (GFI), comparative fit indez (CFI), normed fit index (NFI) e non normed fit index (NNFI).

Os índices para o modelo inicial demonstram que o modelo apresenta bons índices de ajuste, pois todos os valores encontravam-se dentro dos limites de referências (HAIR, 2005), com exceção do CFI, NFI e NNFI. $0 \chi^{2}$ foi de 264,631 , significativo a 0,$001 ; \chi^{2} / \mathrm{gl}$ foi de 1,75 , estando dentro do intervalo considerado como ideal; o RMSEA foi de 0,056, estando abaixo dos 0,08 recomendados como limite máximo, o RMR 
encontrado foi de 0,097 , GFI de 0,894 , CFI de 0,846 ; NFI de 0,714 e NNFI de 0,829 , sendo que todos estes índices devem apresentar valores superiores a 0,90 . 0 root mean square error of aproximation encontra-se abaixo do limite máximo de 0,08 (RMSEA= $0,056)$ o que indica que o modelo se ajusta a uma população e não só a amostra usada para a estimação (Hair, et al., 2005) e o qui-quadrado normalizado foi de 1,75, bem abaixo do limite aceitável de 5,00, o que demonstra que os valores estimados foram próximos do ideal e pode-se afirmar que o ajuste global do modelo foi muito bom.

Os resultados apontam que o modelo é identificado, ou seja, capaz de gerar estimativas únicas. 0 fato de não haver variâncias-erro negativas, coeficientes padronizados com valores acima de 1,00 e erros padronizados muito grandes, apontam que o modelo não apresenta estimativas discrepantes.

Outra etapa da AFC é a avaliação da matriz de resíduos normalizados que, com a significância de 0,05 adotada, no máximo $5 \%$ dos dados pode estar fora do intervalo $-2,58$ a 2,58. Dos 179 resíduos normalizados encontrados, 20 estavam fora desse intervalo, o que aponta que apenas $11,17 \%$ dos resíduos não seguiram essa regra, outro dado que atesta o bom ajuste do modelo.

Apesar de os índices encontrados serem satisfatórios, buscou-se um refinamento do modelo. Analisando-se os resultados dos outputs fornecidos pelo software Amos, duas alterações apareceram acrescentando resultados significativos de melhoria. A primeira alternativa era a extração da variável A10, que possuía um coeficiente de determinação bem inferior aos demais $(0,187)$ e da variável A6 $(0,389)$. Além disso, o modelo poderia ser melhorado com o acréscimo de covariâncias entre os construtos desejo de controle e desejo de status (e23 e e20).

Num primeiro momento, optou-se pela solução menos radical, o acréscimo de covariância entre controle e status, porém essa alternativa criou um modelo não válido, com o surgimento de variância negativa. Dessa forma, optou-se pela exclusão das variáveis com menores coeficientes de determinação. Assim, primeiramente foi excluída apenas a variável A10, surgindo o Modelo 2, o que acarretou uma pequena melhora nos índices, tornando o modelo ainda mais ajustado. Posteriormente, o item A6 também foi excluído, ocorreu a alteração dos índices, alguns para valores mais próximos do ideal e outros para valores que pioravam o ajuste do modelo.
Os três modelos testados apresentam índices satisfatórios, sendo que a exclusão das variáveis A10 e A6 gerou uma melhora nos índices CFI, NFI e NNFI. Conforme argumentam Hair et al. (2005), a eliminação de itens que prejudiquem o desempenho de um modelo é uma das alternativas mais comuns para a melhoria de ajuste do modelo, porém é importante ter em mente que o objetivo da análise fatorial confirmatória é o de testar uma teoria e, portanto, deve-se realizar uma análise criteriosa no momento da exclusão. Ainda segundo os autores, é importante satisfazer as exigências de identificação estatística e, se os dois itens com cargas fatoriais baixas fossem eliminados do modelo, o construto amoralidade ficaria apenas com três itens, ou seja, o número mínimo sugerido para a realização de equações estruturais. Assim, em relação ao item A6, a melhoria obtida no ajuste do modelo não é suficiente para justificar a sua exclusão. Diante do exposto, considera-se que o melhor modelo é o Modelo 2, agora chamado de Modelo Final (Figura 1).

Figura 1 - Resultado da AFV para o Modelo Refinado da Escala MPS

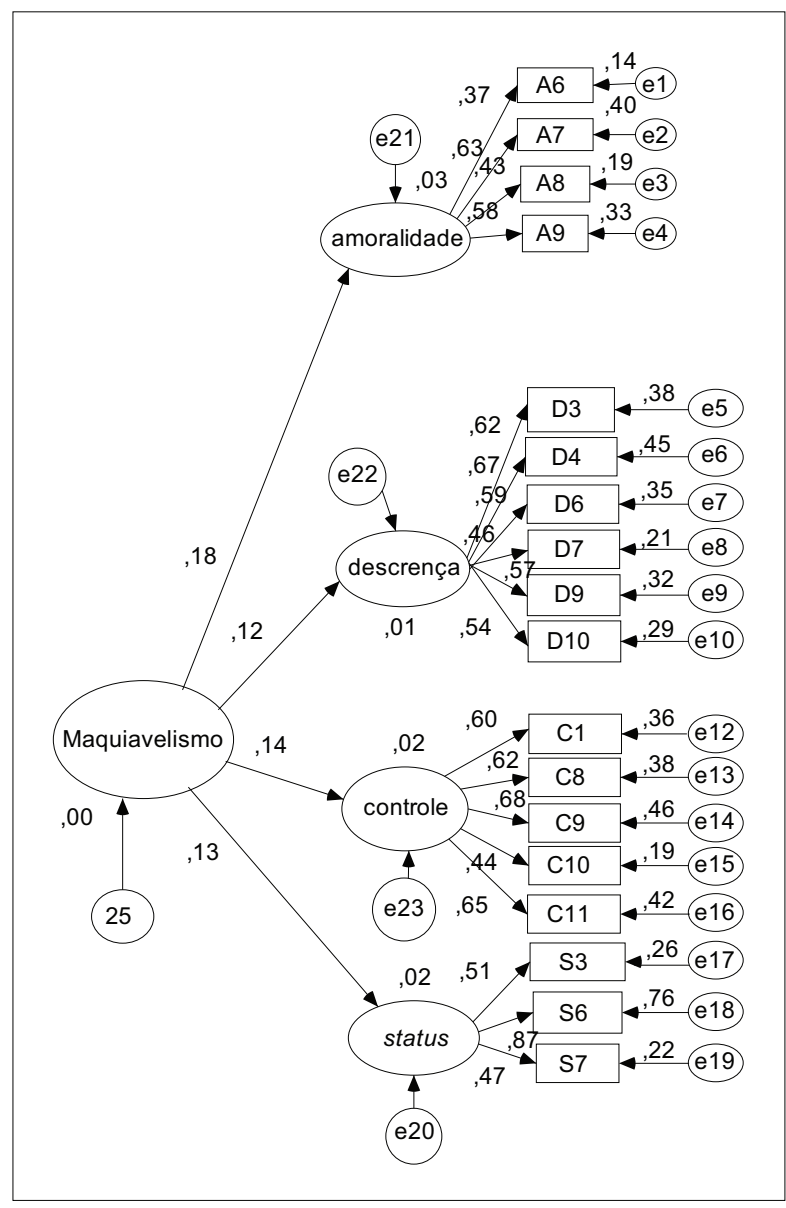

Fonte: Dados da pesquisa. 
Dessa forma, comparando-se os resultados do Modelo Inicial (Modelo 1, originado pela Análise Fatorial Exploratória) e o Modelo Final, no que diz respeito à avaliação do ajuste geral do modelo, o RMSE alterou-se de 0,056 para 0,057, ficando ainda bem abaixo do limite aceitável, o qui-quadrado normalizado continua dentro do intervalo aceitável, aproximando-se mais do valor de 2,00 (intervalo ideal), sendo que se alterou de 1,75 para 1,77 . Os outros índices elevaram-se (GFI de 0,894 para 0,902; CFI de 0,846 para 0,860 ; NFI de 0,714 para 0,734 e NNFI de 0,829 para 0,840 ), porém os índices CFI, NFI e NNFI, apesar de estarem muito próximos do limite ideal, ainda permaneceram abaixo do 0,90.

Sobre a avaliação da matriz de resíduos normalizados, dos 172 resíduos normalizados encontrados, 13 estavam fora desse intervalo, ou seja, apenas 7,6\% de resíduos ficaram fora do intervalo $-2,58$ a 2,58, muito próximo dos $5 \%$ ideais, esta informação corrobora com a melhora obtida no segundo modelo.

A última análise sobre a AFC foi a verificação dos coeficientes de determinação apresentadas pelo diagrama de caminho (Tabela 3). As variáveis $A 7(0,63)$ e A9 $(0,58)$ são as que apresentam maior relação com a variável latente amoralidade, sendo que a variável amoralidade apresenta uma relação de 0,18 com o maquiavelismo, ou seja, $18 \%$ da variância do maquiavelismo são explicadas pelos itens do construto amoralidade. A variável latente descrença teve uma maior relação com o item D4 $(0,67)$ e com o item D3 $(0,62)$ e a menor relação é com o item D7 $(0,46)$, destaca-se, ainda que esse foi o construto com valores mais elevados de coeficientes, sendo que o construto descrença contribui com a explicação de $12 \%$ da variância total encontrado do maquiavelismo. As maiores relações da variável latente controle são com o item C8 $(0,68)$ e C11 $(0,65)$ e a menor relação ocorre com o item C10 $(0,44)$ e este construto explica $14 \%$ da variância do maquiavelismo. Por fim, quanto ao status, o item de relação mais forte é o S6 $(0,87)$ e o mais fraco o S7 $(0,47)$, sendo que o status responde por $13 \%$ da variância do maquiavelismo.

Assim, as análises anteriores demonstram claramente que ambos os modelos testados têm índices de ajuste considerados como bons, porém os índices do segundo modelo - chamado de Modelo Refinado - apresentam índices ainda melhores. Portanto, a adaptação do modelo proposto por Dahling,
Whitaker e Levy (2009) para o contexto nacional resultou na escala de mensuração do maquiavelismo apresentada na Tabela 3, composta de 18 itens divididos em quatro construtos. E, conforme os resultados encontrados neste estudo, os construtos mais importantes para a mensuração do maquiavelismo são, respectivamente: amoralidade (18\%), desejo de controle (14\%), desejo de status (13\%) e descrença nos outros (12\%).

\section{Tabela 3 - Escala MPS adaptada para a realidade brasileira}

\begin{tabular}{l} 
Construto Amoralidade \\
\hline Não há desculpas para enganar outra pessoa (I). \\
Eu só presto atenção no que as pessoas dizem para descobrir \\
se elas sabem algo que possa me prejudicar. \\
A única boa razão para falar com os outros é obter informa- \\
ções que possam me beneficiar. \\
Eu estou disposto a não ser ético se isso me ajudar a ter \\
sucesso. \\
Construto Descrença nos Outros
\end{tabular}

Eu prefiro trabalhar sozinho do que depender do desempenho dos outros.

As pessoas só são motivadas por objetivos pessoais.

Quando tenho uma boa ideia, não conto aos outros para evitar que a roubem.

Eu gosto de dividir meus planos e ideias com outras pessoas (I).

Membros de equipe puxam o tapete uns dos outros para conseguir destaque.

Se eu mostrar alguma fraqueza no trabalho, os outros irão tirar vantagens disso.

\section{Construto Desejo de Controle}

Eu acho que medo e ameaças são coisas necessárias para motivar as pessoas a fazerem o que eu quero.

As ações dos outros influenciam constantemente minhas chances de sucesso.

Em minha vida, eu controlo o curso dos eventos.

Eu gosto de ter habilidade de controlar a situação.

Eu determino o que acontece em minha vida.

\section{Construto Desejo de Status}

Eu acredito que a maioria das pessoas gosta de exibir seu próprio sucesso.

Algum dia eu quero ser rico e poderoso.

0 desenvolvimento das pessoas é um dos meus objetivos mais importantes (I).

Legenda: (I) itens com análises inversas.

Fonte: Dados da pesquisa. 


\section{Conclusões}

Quanto ao objetivo deste estudo, observa-se que, assim como em várias outras áreas da administração, pesquisas americanas e europeias são tomadas como base para estudos no Brasil e suas escalas são utilizadas sem a preocupação de uma avaliação adequada e da validação de um modelo desenvolvido e validade em outra realidade e cultura. E, os resultados desta pesquisa demonstraram que esse pode ser um grande equívoco, pois o modelo validado por Dahling, Whitaker e Levy (2009) foi diferente do validado na presente pesquisa, o que reforça a necessidade de validação de todas as escalas que são desenvolvidas no exterior e aplicadas no Brasil.

É importante destacar que este trabalho tem uma série de limitações. Em primeiro lugar, pelo fato de utilizar uma escala de mensuração nova e que ainda não foi devidamente reaplicada e testada estatisticamente. Outra limitação é o fato de que, pela escassez de estudos que utilizaram a MPS, não é possível fazer relações dos resultados deste estudo com outras pesquisas. E, por fim, a principal limitação encontrada foi a de que não se podem realizar comparações diretas com o modelo validado por Dahling, Whitaker e Levy (2009), pois, com a amostra pesquisada, a AFC para o Modelo Original obteve variância negativa e várias análises ligadas à correlações não puderam ser realizadas. Os poucos resultados obtidos demonstraram índices semelhantes com os do artigo original.

Diante das limitações, surge a necessidade de algumas novas pesquisas sobre o tema que, em suma, poderiam: revalidar a escala MPS para a realidade brasileira; aplicar à escala outros ambientes organizações para que se pudessem fazer comparações; relacionar o maquiavelismo com outros construtos dos estudos organizacionais para que se tenha um panorama do nível de maquiavelismo nas organizações brasileiras e suas interações.

Ao término deste estudo, foi possível identificar que o construto maquiavelismo apresenta uma série de relações com diversos temas dos estudos organizacionais, sendo que as principais relações referem-se à liderança, influências, satisfação, competição, etc. As relações teóricas já se encontram em um patamar avançando, porém observou-se, ao longo da pesquisa bibliográfica, que a utilização do construto Maquiavelismo ainda é incipiente no Brasil (não foi encontrado nenhum artigo sobre maquiavelismo organizacional em ambientes nacionais, na última década) e que várias outras conexões precisam ser realizadas e testadas como, por exemplo, as influências de comportamentos maquiavélicos para a confiança, as políticas organizacionais, a gestão do poder e da ética nas organizações. Em suma, a importância do maquiavelismo para os estudos organizacionais é inegável e torna-se necessário incentivar pesquisas nessa área.

\section{Referências}

Anderson, J., Gerbing, D., \& Hunter, J. (1987). On the assessment of unidimensional measurement: Internal and external consistency, and overall consistency criteria. Journal of Marketing Research, 24(4), 432-437.

Christie, S., \& Geis, F. (1970). Studies in Machiavellianism. San Diego: Academic Press.

Dahling, J., Whitaker, B., \& Levy, P. (2009). The development and validation of a new machiavellianism scale. Journal of Management, 35, 219-257.

Deluga, R. (2001). American presidential Machiavellianism: Implications for charismatic leadership and rated performance. Leadership Quarterly, 12, 339-363.

Fehr, B., Samson, D., \& Paulhus, D. R. (1992). The construct of Machiavellianism: Twenty years later. In C. D. Spielberger \& J. N. Butcher (Ed.). Advances in personality assessment (pp. 77-116). Hillsdale, NJ: Erlbaum.

Gable, M., \& Dangelo, F. (1994). Locus of control, Machiavellianism, and managerial job performance. The Journal of Psychology, 128(5), 599-608.

Gable, M., \& Topol, M. (1991). Machiavellian managers: Do they perform better. Journal of Business Psychology, 5(3), 355-384.

Gable, M., \& Topol, M. (1998). Machiavellianism and the department store. Journal of Retailing, 64(1), 68-84.

Geis, F., \& Moon, T. (1981). Machiavellianism and deception. Journal of Personality and Social Psychology, 41, 766-775.

Gunnthorsdottir, A., Mccabe, K., \& Smith, V. (2002). Using the Machiavellianism instrument to predict trustworthiness in a bargaining game. Journal of Economic Psychology, 23(1), 49-66. 
Hair, J. F., Anderson, R. E., Tatham, R. L., \& Black, W. C. (2005). Análise multivariada de dados. Porto Alegre: Bookman.

Liu, C. (2008). The relationship between Machiavellianism and knowledge sharing willingness. Journal of Business Psychology, 22(3), 233-240. doi: 10.1007/ s10869-008-9065-1

Lopes, H. E. (2005). Abrindo a caixa preta: Considerações sobre a utilização da análise fatorial confirmatória nas pesquisas em administração. E \& G Economia e Gestão, 5(11), 78-97.

Malhotra, N. (2001). Pesquisa de marketing: Orientação aplicada. Porto Alegre: Bookman.

Pestana, M., \& Gageiro, J. (2000). Análise de dados para ciências sociais: A complementaridade do SPSS. Lisboa: Gráfica Rolo e filhos.

Ricks, J., \& Fraedrich, J. (1999). The paradox of Machiavellianism: Machiavellianism may mala for productive sales but poor management reviews. Journal of Business Ethics, 20, 197-205.

Ross, W., \& Robertson, D. (2003). A typology of situational factors: Impact on salesperson decision-making about ethical issues. Journal of Business Ethics, 46, 213-234.
Sakalaki, P., Kanellaki, S., \& Richardson, C. (2009). Is a Manipulator's Externality Paradoxical? The Relationship Between Machiavellianism, Economic Opportunism, and Economic Locus of Control. Journal of Applied Social Psychology, 39(11), 2591-2603.

Schepers, D. (2003). Machiavellianism, profit, and the dimensions of ethical judgment: A study of impact. Journal of Business Ethics, 42, 339-352.

Shomenand, A., \& Raon, H. (2009). Machiavellianism in public accountants: Some additional Canadian evidence. Business Ethics: A European Review, 18, 315-328.

Spector, P. E. (1992). Summated rating scale construction: An introduction. London: Sage.

Tang, T., Chen, Y. (2008). Intelligence vs. wisdom: The love of money, Machiavellianism, and unethical behavior across college major and gender. Journal of Business Ethics, 82, 1-26.

Vleeming, R. G. (1979). Machiavellianism: A preliminary review. Psychological Reports, 44(1), 295-310. doi: 10.2466/pr0.1979.44.1.295

White, G. (1984). Comparison of four jealousy scales. Journal of Research in Personality, 18(2), 121125. doi.org/10.1016/0092-6566(84)90024-2

Zook, A. (1985). On measurement of Machiavellianism. Psychological Reports, 57, 982-1003. 